
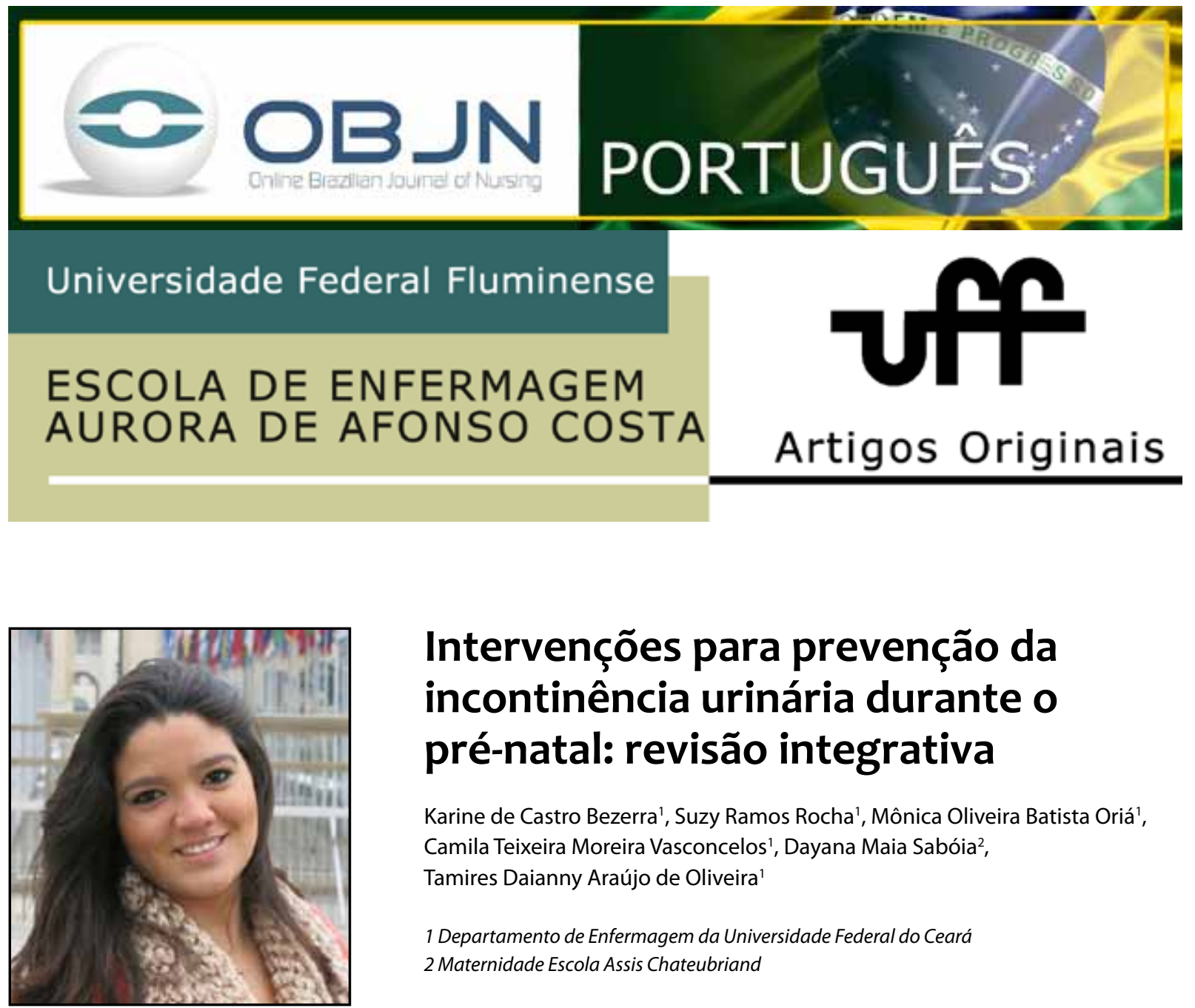

\title{
Intervenções para prevenção da incontinência urinária durante $o$ pré-natal: revisão integrativa
}

\author{
Karine de Castro Bezerra', Suzy Ramos Rocha', Mônica Oliveira Batista Oriá', \\ Camila Teixeira Moreira Vasconcelos ${ }^{1}$, Dayana Maia Sabóia², \\ Tamires Daianny Araújo de Oliveira ${ }^{1}$ \\ 1 Departamento de Enfermagem da Universidade Federal do Ceará \\ 2 Maternidade Escola Assis Chateubriand
}

\section{RESUMO}

Objetivo: avaliar as evidências disponíveis na literatura sobre intervenções para prevenir incontinência urinária em gestantes durante o acompanhamento pré-natal. Método: revisão integrativa da literatura (RI). A busca foi realizada em outubro de 2014 nas bases de dados LILACS, MEDLINE, CINAHL e Cochrane. Onze artigos compuseram a amostra da RI. Resultados: todos os artigos foram publicados no idioma inglês; sete deles identificados na base de dados PubMed, e quatro na Cochrane. A maior parte dos trabalhos avaliados utilizaram intervenções cognitivas e comportamentais, sendo o treino da musculatura do assoalho pélvico o principal tratamento para prevenir e tratar a incontinência urinária durante a gestação. Conclusão: os resultados obtidos podem cooperar para o aperfeiçoamento da assistência da mulher no ciclo gravídicopuerperal.

Descritores: Incontinência Urinária; Cuidado Pré-natal; Enfermagem. 


\section{INTRODUÇÃO}

A incontinência urinária (IU) feminina é um importante problema de saúde pública, seja por sua elevada prevalência ou pelos impactos físico, psíquico e social na vida da mulher ${ }^{(1,2)}$. Em 2012, o comitê da Internacional Continence Society (ICS) atualizou as terminologias e definições das disfunções do trato urinário inferior. Assim, a ICS define IU como a queixa de perda involuntária de urina, cuja forma mais comum nas mulheres é a incontinência urinária de esforço (IUE), considerada a mais prevalente nos anos reprodutivos ${ }^{(3)}$. Este tipo de IU é caracterizado como a perda involuntária de urina durante esforço ou exercício físico ou ao espirrar ou tossir, que pode ocorrer por alterações anatômicas como a hipermobilidade do colo vesical e a deficiência do mecanismo esfincteriano $^{(4)}$.

A etiologia da IUE não se encontra totalmente esclarecida, mas os traumatismos ocorridos no pavimento pélvico durante a gravidez e o parto são sugeridos como os principais fatores de risco ${ }^{(5)}$. Apesar de a IUE ser um problema comum, as estimativas da sua prevalência, obtidas por meio de estudos epidemiológicos, variam consideravelmente, podendo oscilar entre $3,6 \%$ e $15 \%$ antes de engravidar ${ }^{(5)}$ e $28 \%$ e $64 \%$ durante a gravidez ${ }^{(4)}$.

A IUE é de etiologia multifatorial, e a gestação é um fator significante para seu desenvolvimento. A profilaxia é determinante na vida da mulher, pois as estruturas que suportam os órgãos pélvicos necessitam adaptar-se à sobrecarga do peso e à passagem do feto durante o parto vaginal. Para isso, os exercícios perineais e o preparo dos músculos do assoalho pélvico durante o período gestacional são fundamentais para prevenir danos neuromusculares e a IUE ${ }^{(3)}$.

Assim, acreditando que uma oportunidade para a prevenção poderá iniciar-se na consulta de enfermagem no pré-natal, a gravidez é o período ideal para educar a mulher quanto a sua saúde. Nesta fase, ela tem um contanto constante com diversos profissionais da saúde, sendo incentivada a melhorar seu estado físico pela própria gestação(5). Diante desse cenário, surgiu o seguinte questionamento: Quais as intervenções desenvolvidas para gestantes durante o pré-natal com vistas à prevenção da IU?

Ao considerar o enfermeiro um potencial educador que visa promover a saúde, este trabalho teve a finalidade de avaliar as evidências disponíveis na literatura sobre intervenções para prevenir IU em gestantes durante o acompanhamento pré-natal.

\section{MÉTODO}

Para o alcance do objetivo proposto selecionou-se como método de pesquisa a revisão integrativa, delimitando-se as seguintes etapas percorridas: estabelecimento da hipótese e objetivos da revisão integrativa, desenvolvimento de critérios de inclusão e exclusão de artigos (seleção da amostra), definição das informações a serem extraídas dos artigos selecionados, análise dos resultados, discussão e apresentação dos resultados e, por fim, a apresentação da revisão(6).

Para seleção dos artigos foram utilizadas as seguintes bases de dados: Literatura Latino-Americana e do Caribe em Ciências da Saúde (LILACS), MEDLINE, Cumulative Index to Nursing and Allied Health Literature (CINHAL) e Cochrane.

Os critérios de inclusão, trabalhos publicados nos idiomas português, inglês ou espanhol divulgados no período de 2005 a 2015 e que retratassem intervenções utilizadas durante o pré-natal para prevenir a IU. Excluíram-se 
artigos que não contemplassem a questão norteadora da pesquisa, resumos e anais de congressos, comentários, artigos de revisão, editoriais, opiniões e relatórios e pesquisas sobre outras patologias ou estudos com detaIhamento metodológico incompleto.

Os dados foram coletados no mês de outubro de 2014, com os seguintes descritores controlados: Incontinência Urinária (Urinary Incontinence), Cuidado Pré-natal (Prenatal Care) e Enfermagem (Nursing), combinados em cada base de dados de acordo com a necessidade para assegurar ampla busca.

Para a categorização dos dados, utilizou-se um instrumento adaptado de Mendes et $\mathrm{al}^{(6)}$, que contempla identificação do artigo original, características metodológicas do estudo e avaliação do rigor metodológico, das intervenções mensuradas e dos resultados encontrados.

Para a análise e posterior síntese dos artigos que atenderam os critérios de inclusão, construiu-se um quadro sinóptico também $\operatorname{adaptado}^{(7)}$ para esse fim, com nome da pesquisa e dos autores, intervenção estudada, resultados, recomendações e conclusões.

Após analisadas, as intervenções foram categorizadas em comportamentais, cognitivas ou sociais ${ }^{(8)}$. As intervenções comportamentais propõem estímulos associados à adesão ao tratamento (exemplo: lembretes). Cognitivas fornecem novas informações, educam mulheres sobre os tipos de tratamento e esclarecem possíveis conceitos errôneos. Já as sociais utilizam peers counselors (pessoas da comunidade treinadas para oferecer informações) ou profissionais com vistas a aumentar a adesão ao tratamento ${ }^{(8)}$.

As informações obtidas foram apresentadas por meio de tabelas e a discussão dos dados obtidos foi feita de forma descritiva, possibilitando ao leitor a avaliação da aplicabilidade da revisão integrativa elaborada.

\section{RESULTADOS}

Após a aplicação dos critérios de inclusão e exclusão, a amostra final consistiu de 11 artigos. Sete deles foram identificados na base de dados PubMed, e quatro na Cochrane. Apenas dois artigos foram publicados em revistas de enfermagem, enquanto nove estavam em revistas médicas. Todos os artigos foram publicados em revistas internacionais, em inglês. A Figura 1 retrata o delineamento utilizado para a seleção dos estudos.

Figura 1 - Fluxograma do processo de seleção de artigos inclusos na revisão integrativa. Fortaleza, 2014

Artigos identificados nas bases de dados

LILACS, Pubmed, Cinahl e Cochrane $(n=732$

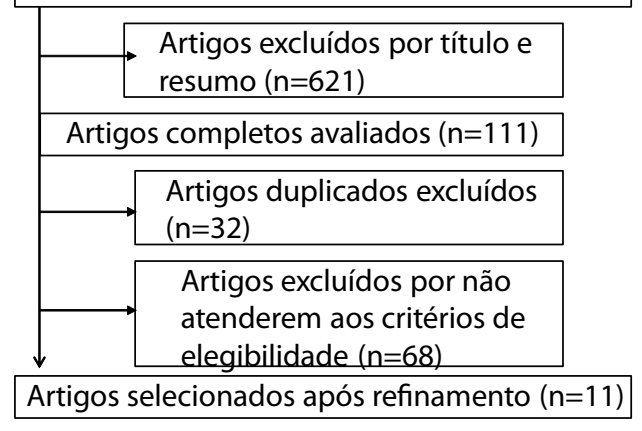

Fonte: elaboração dos autores, 2014.

Com relação ao nível de evidência, sete estudos apresentaram nível de evidência II, ou seja, ensaios clínicos controlados randomizados (ECCR); um estudo primário apresentou nível de evidência III, com delineamento quase experimental; dois estudos com nível de evidência IV, coorte prospectivos; e um estudo com nível de evidência $\mathrm{VI}$, com metodologia descritiva. $\mathrm{O}$ Quadro 1 lista os detalhes dos estudos incluídos na revisão.

No tocante à avaliação das intervenções utilizadas, a maioria dos grupos intervenção utilizaram programas de treinamento da musculatura do assoalho pélvico durante o pré-natal, comparando com os cuidados habituais. 
O tempo de realização das intervenções foi de 12 meses, em média. $O$ início do treinamento ocorreu em gestantes de 20 a 36 semanas de gestação e a maior parte das intervenções foram categorizadas em cognitivas e comportamentais. A síntese dos estudos primários incluídos na revisão, quanto à intervenção realizada e os principais desfechos encontram-se no Quadro 2.

Quadro 1 - Detalhes dos estudos incluídos na revisão. Fortaleza, 2014.

\begin{tabular}{|l|l|l|l|l|}
\hline \multicolumn{1}{|c|}{ Estudo } & Desenho & $\begin{array}{l}\text { Nível } \\
\text { de evi- } \\
\text { dência }\end{array}$ & $\begin{array}{c}\text { Amos- } \\
\text { tra }\end{array}$ & Ano \\
\hline $\begin{array}{l}\text { Whitford et } \\
\text { al(8) }\end{array}$ & $\begin{array}{l}\text { Coorte } \\
\text { prospectivo }\end{array}$ & IV & N=289 & 2006 \\
\hline Stafne et al(9) & ECCR* & II & $\mathrm{N}=855$ & 2012 \\
\hline $\begin{array}{l}\text { Bo e Haaks- } \\
\text { tad(10) }\end{array}$ & ECCR & II & $\mathrm{N}=105$ & 2011 \\
\hline $\begin{array}{l}\text { Miquelutti et } \\
\text { all(11) }\end{array}$ & ECCR & II & $\mathrm{N}=197$ & 2013 \\
\hline $\begin{array}{l}\text { Eliasson et } \\
\text { al(12) }\end{array}$ & $\begin{array}{l}\text { Coorte } \\
\text { prospectivo }\end{array}$ & IV & $\mathrm{N}=665$ & 2004 \\
\hline $\begin{array}{l}\text { Sangsawang e } \\
\text { Serisathien(13) }\end{array}$ & $\begin{array}{l}\text { Quase-ex- } \\
\text { perimental }\end{array}$ & III & $\mathrm{N}=66$ & 2011 \\
\hline $\begin{array}{l}\text { Morkved et } \\
\text { al(14) }\end{array}$ & ECCR* & II & $\mathrm{N}=301$ & 2003 \\
\hline $\begin{array}{l}\text { Butterfield et } \\
\text { al(15) }\end{array}$ & Descritivo & VI & $\mathrm{N}=225$ & 2007 \\
\hline Reilly et al(16) & ECCR* & II & $\mathrm{N}=268$ & 2002 \\
\hline Low et al(17) & ECCR* & II & $\mathrm{N}=249$ & 2013 \\
\hline Agur et al(18) & ECCR* & II & $\mathrm{N}=230$ & 2008 \\
\hline
\end{tabular}

Fonte: elaboração dos autores, 2014.

*ECCR=Ensaio Clínico Controlado Randomizado

\section{DISCUSSÃO}

Como demonstrado nesta revisão, há necessidade urgente de se realizar atividades educativas e preventivas com a bexiga durante a gestação. Neste sentido, a consulta de enfermagem pré-natal é uma excelente ferramenta que identifica os fatores de risco para IU índice de massa corpórea elevado; hábitos intestinais inadequados; presença de constipação; sintomas urinários - episódios de perda involuntária de urina ao tossir, espirrar, agachar, levantar peso (na gestação atual ou em anteriores) -; e número de gestações e paridade ${ }^{(2)}$.

O enfermeiro deve investigar os sintomas urinários, já que muitas gestantes ou mesmo puérperas não relatam suas queixas por acreditarem que a perda de urina é normal e passageira ou tem relação com o processo de gestação. Ao toque vaginal, também é preciso avaliar a força dos músculos do assoalho pélvico. Dessa forma, tem-se o conhecimento da consciência desses músculos pela mulher. Sendo esse achado, um dos principais dados a serem registrados no sentido de prevenção da IUE. A partir dessas informações, podem-se orientar as gestantes quanto ao risco de ganho de peso, de mau funcionamento do intestino e sua relação com a IU $U^{(9)}$.

Desde 1948, Arnold Kegel enfatizou o valor dos exercícios de fortalecimento perineal na recuperação da função dos músculos do assoalho pélvico em mulheres com IUE. Estes exercícios têm base no preceito de que os movimentos voluntários repetidos proporcionam aumento da força muscular ${ }^{(20)}$. Entretanto, para que as muIheres assumam um comportamento preventivo em saúde, é preciso um trabalho de conscientização e educação por meio de informações, mostrando as mudanças do assoalho pélvico que podem ocorrer na gestação. É preciso ensinar as gestantes os exercícios de Kegel e sua eficácia no fortalecimento dessa musculatura.

Nesse sentido, Kocaöz et all (2013), com vistas à educação em saúde, afirmam que o profissional da saúde deve encorajar as mulheres a adotar e manter padrões de vida sadios. Sendo assim, o enfermeiro tem papel destacado na equipe multiprofissional, agindo como um elemento catalizador das mudanças necessárias para a melhor qualidade de vida de suas clientes. Um programa de exercícios para os músculos do 
Quadro 2 - Síntese dos estudos primários incluídos na revisão. Fortaleza, 2014.

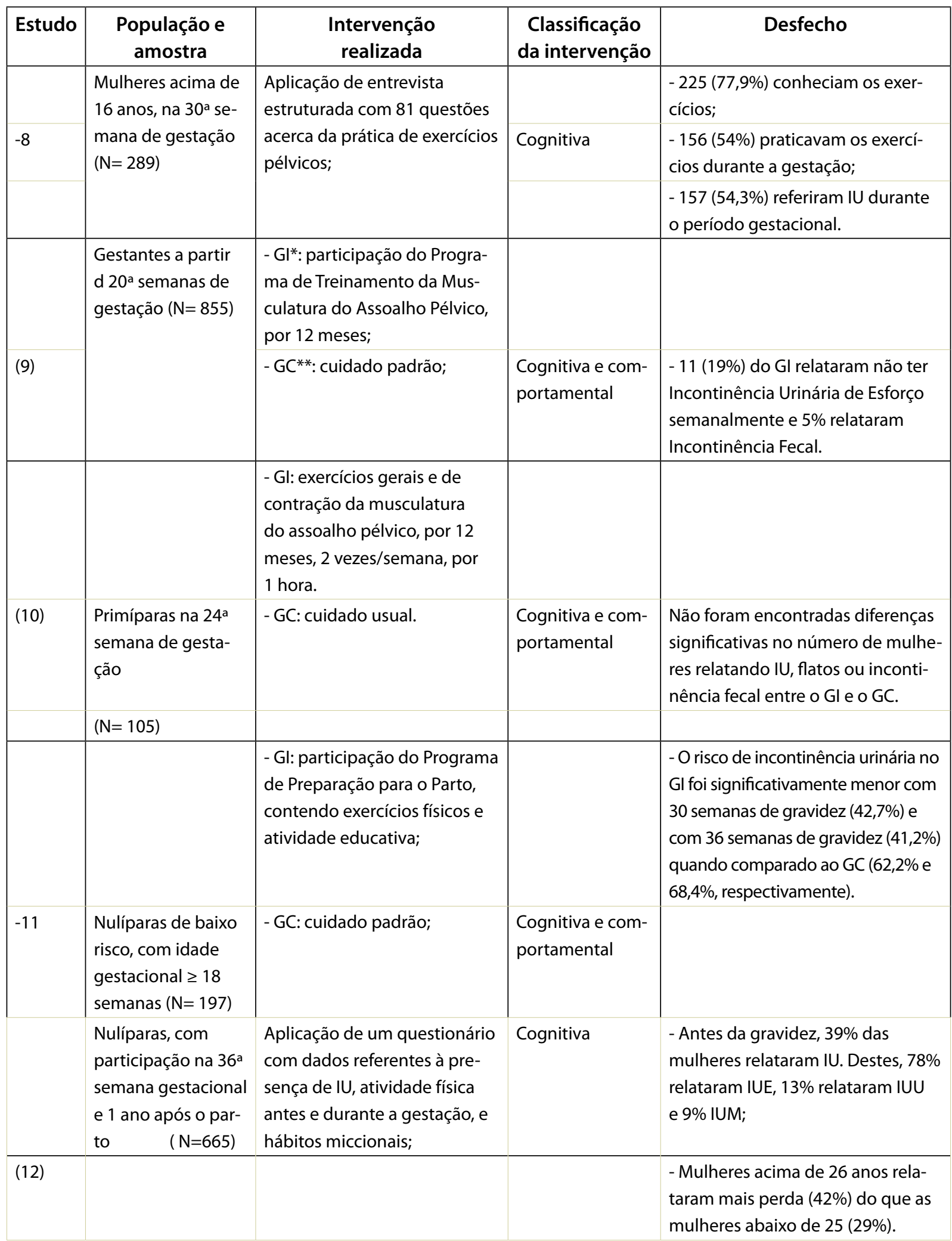




\begin{tabular}{|c|c|c|c|c|}
\hline & $\begin{array}{l}\text { Gestantes com } \\
\text { IUEף na } 20^{\mathrm{a}}-30^{\mathrm{a}} \\
\text { semana gestacional } \\
(\mathrm{N}=66)\end{array}$ & $\begin{array}{l}\text { - GE: participação no Progra- } \\
\text { ma de exercício da musculatu- } \\
\text { ra do assoalho pélvico }\end{array}$ & & $\begin{array}{l}\text { - As mulheres do grupo expe- } \\
\text { rimental tiveram frequência e } \\
\text { volume de perda de urina, e escore } \\
\text { da gravidade da IUE percebido } \\
\text { após a participação, significativa- } \\
\text { mente menor do que os do grupo } \\
\text { de controle. }\end{array}$ \\
\hline \multirow[t]{2}{*}{ (13) } & & - GC: cuidado padrão; & $\begin{array}{l}\text { Cognitiva e com- } \\
\text { portamental }\end{array}$ & \\
\hline & $\begin{array}{l}\text { Gestantes a partir } \\
\text { da } 20^{\text {a }} \text { semana ges- } \\
\text { tacional }(\mathrm{N}=301)\end{array}$ & $\begin{array}{l}\text { - Gl: participação do Programa } \\
\text { de Treinamento da Muscula- } \\
\text { tura do Assoalho Pélvico, por } \\
12 \text { meses; }\end{array}$ & & $\begin{array}{l}\text { - Significativamente menos mu- } \\
\text { lheres no GI referiram IU: } 48 \text { de } 148 \\
\text { (32\%) versus } 74 \text { de } 153(48 \%) \text { em } \\
\text { gestantes de } 36 \text { semanas ( } p=0,007) \\
\text { e } 29 \text { de } 148 \text { ( } 20 \% \text { ) versus } 49 \text { de } 153 \\
(32 \%) \text { com } 3 \text { meses após o parto; }\end{array}$ \\
\hline \multirow[t]{2}{*}{ (14) } & & - GC: cuidado padrão; & $\begin{array}{l}\text { Cognitiva e com- } \\
\text { portamental }\end{array}$ & $\begin{array}{l}\text { - Força da musculatura do assoalho } \\
\text { pélvico foi significativamente } \\
\text { maior no grupo intervenção com } \\
36 \text { semanas de gestação }(p=0,008) \\
\text { e } 3 \text { meses após o parto }(p=0.048) \text {. }\end{array}$ \\
\hline & $\begin{array}{l}\text { Parteiras presta- } \\
\text { doras de service } \\
\text { pré-natal, perinatal } \\
\text { e pós-natal ( } \mathrm{N}= \\
225) \text {. }\end{array}$ & $\begin{array}{l}\text { Aplicação de um instrumento } \\
\text { que continha questões sobre } \\
\text { período pré-natal, perinatal e } \\
\text { pós-natal; bem como fatores } \\
\text { de risco associados à IU. }\end{array}$ & & $\begin{array}{l}\text { - Existiam } 131 \text { (58,2\%) parteiras } \\
\text { prestando serviço pré-natal, } 168 \\
\text { (79\%) cuidando das mulheres no } \\
\text { intraparto e } 186(86,9 \%) \text { prestando } \\
\text { cuidados no período pós-natal. }\end{array}$ \\
\hline \multirow[t]{2}{*}{ (15) } & & & Cognitiva & $\begin{array}{l}\text { - Apenas } 106(64,6 \%) \text { já avaliaram } \\
\text { guidelines, mas apenas } 89,6 \% \text { des- } \\
\text { tas ( } n=69) \text { utilizavam o guideline } \\
\text { para guiar a prática. }\end{array}$ \\
\hline & $\begin{array}{l}\text { Primigestas, com } \\
20 \text { semanas de } \\
\text { gestação }(N=268)\end{array}$ & $\begin{array}{l}\text { - Gl: orientações quanto à } \\
\text { pratica de exercícios com } \\
\text { repetições de } 8 \text { contrações } \\
\text { com duração de } 6 \text { segundos, } \\
\text { com dois minutos de repouso } \\
\text { entre as repetições, duas } \\
\text { vezes ao dia }\end{array}$ & & \\
\hline (16) & & - GC: cuidado padrão; & $\begin{array}{l}\text { Cognitiva e com- } \\
\text { portamental }\end{array}$ & $\begin{array}{l}\text { - Apenas } 19,2 \% \text { das gestantes do } \\
\text { Gl referiram incontinência de esfor- } \\
\text { ço pós-parto, comparados a } 32,7 \% \\
\text { do grupo controle; }\end{array}$ \\
\hline \multirow[t]{2}{*}{ (17) } & $\begin{array}{l}\text { Gestantes a partir } \\
\text { da } 20^{\text {a }} \text { semana ges- } \\
\text { tacional }(\mathrm{N}=249)\end{array}$ & Quatro grupos: & & \\
\hline & & $\begin{array}{l}\text { - (1) cuidados de rotina com } \\
\text { expulsão treinada ou dirigida }\end{array}$ & $\begin{array}{l}\text { Cognitiva e com- } \\
\text { portamental }\end{array}$ & $\begin{array}{l}\text { - Não houve diferença estatística } \\
\text { na incidência de incontinência } \\
\text { pós-parto baseada com o método } \\
\text { de expulsão (valor de } p=0,57 \text { ) ou } \\
\text { em combinação com massagem } \\
\text { perineal pré-natal (valor } P=0,57 \text { ). }\end{array}$ \\
\hline
\end{tabular}




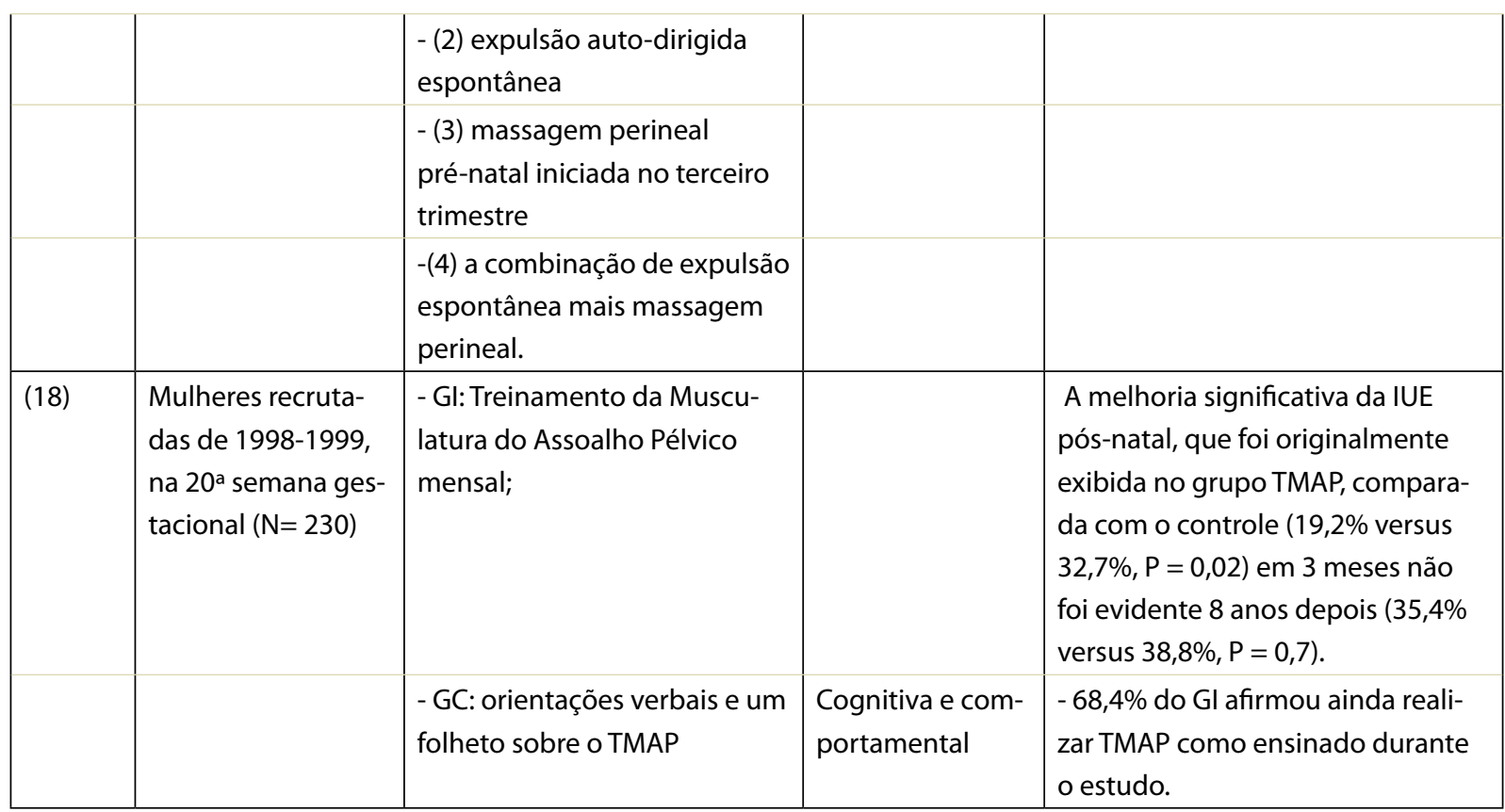

GI*: Grupo intervenção

$G C^{* *}$ : Grupo controle

IUEף: Incontiência Urinária de Esforço

Fonte: elaboração dos autores, 2014.

assoalho pélvico pode ensinar às mulheres a função desses músculos no controle do mecanismo da continência urinária, pois o aumento da força e da resistência muscular auxilia na sustentação da bexiga e no fechamento uretral. Isso é de grande importância para mulheres que pretendem engravidar, pois é esperado um declínio da força do assoalho pélvico da $20^{\text {a }}$ semana de gestação até seis semanas após o parto(21).

Propõe-se, então, que as mulheres façam exercícios perineais durante a gestação, considerando-os uma forma de tratamento conservador eficaz para IUE, além de não causar nenhum efeito colateral.

Nesse propósito, pesquisadores aplicaram um programa de treinamento para fortalecimento dos músculos do assoalho pélvico durante as $20^{\mathrm{a}}$ a $35^{\mathrm{a}}$ semanas de gestação e obtiveram uma maior força dos músculos do assoalho pélvico na 6 a semana de puerpério, quando comparado com um grupo controle, mantendo até 12 meses de pós-parto ${ }^{(22)}$. Com base nesses resultados, os autores recomendam exercícios no pré-natal e no puerpério e a mensuração da força dos músculos do assoalho pélvico em mulheres que pretendem engravidar, incentivando a realização de um programa de treinamento.

Entretanto, Reilly et al (2012) não encontraram muito benefício dos exercícios do assoalho pélvico, pois o número de mulheres que relatou IU no pós-parto foi semelhante para o grupo controle $\left(19,2 \%\right.$ contra $32,7 \%$ respectivamente) ${ }^{(23)}$.

No tocante ao tipo de intervenção educativa a ser realizada, a seleção da mesma deve levar em consideração as características especíicicas de cada população ou serviço. Um exemplo disso é a evidência de que gestantes mais velhas se beneficiam mais de intervenções comportamentais com oficinas educativas ${ }^{(12)}$, enquanto que as gestantes mais jovens, das intervenções cognitivas utilizando lembretes ${ }^{(14)}$.

Nesse sentido, para que ocorram mudanças de conhecimento, são necessárias intervenções educativas à população em geral e aos profissionais de saúde generalistas que promovam a reflexão e tragam elucidações sobre a IU, seu 
impacto sobre a qualidade de vida. Dessa forma, se conduz uma assistência adequada a quem busca ajuda.

No presente estudo, as estratégias comportamentais e cognitivas que utilizaram tecnologias educativas como manuais, vídeos, panfletos, lembretes e programas de educação em saúde se mostraram eficazes na expansão de informações e busca de tratamento, demostrando um grande potencial de reprodutibilidade em outras realidades com vistas a melhorar o conhecimento dessas mulheres, com IU ou com fatores de risco bem elucidados.

\section{CONCLUSÃO}

A IUE é uma afecção de etiologia multifatorial, e a gestação é um fator de risco significativo para seu desenvolvimento. Para tanto, há necessidade dos profissionais que trabalham na área da Saúde da Mulher incorporem na consulta do pré-natal a avaliação da força dos músculos do assoalho pélvico, questionem sobre a perda de urina antes e durante a gestação e valorizem as queixas urinárias, atentando para dados como índice de massa corporal elevado e constipação.

Somado a isso, deve-se ensinar à gestante sobre os exercícios perineais de Kegel, pois eles exercem uma função fundamental na prevenção e correção da IU na gestação e no puerpério, e são considerados o único meio de se prevenir a IUE. Os exercícios podem ser iniciados na $20^{a}$ semana e finalizando na $36^{\text {a }}$ semana de gestação, podendo ser realizadas 40 contrações com tempo de sustentação de 10 segundos, diariamente.

A terapia comportamental e o TMAP são estratégias eficazes tanto na prevenção como no tratamento da IU durante o pré-natal. Essas abordagens, quando utilizadas com estratégias cognitivas e comportamentais, potencializam seus resultados.
Tanto as estratégias comportamentais quanto as cognitivas e sociais mostraram efeitos positivos no tratamento e na prevenção da IU durante a gestação; todavia sugere-se que, quando adequado, se utilize a combinação das intervenções para se obter resultado mais eficaz, como demonstrado.

Ressalta-se a importância da potencialização de uma abordagem preventiva da IU, atuando em fatores de riscos modificáveis, para tanto o enfoque multiprofissional faz-se premente com atuação conjunta na busca de resultados positivos.

Embora todos os estudos sejam oriundos da literatura internacional, realizados em gestantes de diferentes culturas, raças e etnias, bem como usuárias de um sistema de saúde diferenciado, todas as intervenções propostas são de fácil reprodutibilidade e adaptação ao contexto das gestantes brasileiras.

Espera-se, com este estudo, incentivar os profissionais de saúde que trabalham com IU a realizarem pesquisas de intervenção, principalmente no nível de atenção primária, utilizando teorias que respaldem o seu uso, bem como desenhos metodológicos com maior nível de evidência, contribuindo, dessa forma, para prática em saúde consolidada e baseada em evidências.

\section{REFERÊNCIAS}

1. Marecki M, Seo J. Perinatal Urinary and Fecal Incontinence. J Perinat Neonat Nurs. 2010; 24(4):330-40.

2. Sangsawang B. Risk factors for the development of stress urinary incontinence during pregnancy in primigravidae: a review of the literature. Eur J Obstet Gynecol Reprod Biol. 2014:178:27-34.

3. Sangsawang B, Serisathien Y. Effect of pelvic floor muscle exercise programme on stress urinary incontinence among pregnant women. J Adv Nurs [internet]. 2012 [cited 2014 nov 13]; 68(9):1997- 
2007. Available from: http://onlinelibrary.wiley. com/doi/10.1111/j.1365-2648.2011.05890.x/pdf

4. Chiarelli $P$, Cockburn J. Promoting urinary continence in women after delivery: randomised controlled trial. BMJ [internet] 2012 [cited 2014 nov 13]; 324:124. Available from: http://www. bmj.com/content/324/7348/1241.full.pdf+html

5. Morkved S, BO K. Prevalence of urinary incontinence during pregnancy and postpartum. International Urogynecol J. 2014; 10:394-98.

6. Souza MT, Silva MD, Carvalho R Integrative review: what is it? How to do it? einstein. 2010; 8(1 Pt 1):102-106

7. Galvão TF, Pereira MG. Revisões sistemáticas da literatura: passos para sua elaboração. Epidemiol. Serv. Saúde. 2014 23(1):183-184.

8. Vasconcelos CTM, Damasceno MMC, Lima FET, Pinheiro AKB. Integrative review of the nursing interventions used for the early detection of cervical uterine cancer. Rev. Latino-Am. Enfermagem [internet] 2011 [cited 2014 oct 10]; 19:8. Available from: http://www.scielo.br/pdf/rlae/ v19n2/28.pdf.

9. Whitford HM, Alder B, Jones M. A cross-sectional study of knowledge and practice of pelvic floor exercises during pregnancy and associated symptoms of stress urinary incontinence in North-East Scotland. Midwifery 2007, Jun; 23(2):204-17. [Included in the review]

10. Stafne SN, Salvesen KA, Romundstad PR, Torjusen $\mathrm{IH}$, Mørkved S. Does regular exercise including pelvic floor muscle training prevent urinary and anal incontinence during pregnancy? A randomized controlled trial. BJOG [internet] 2012 [cited 2014 oct 10]; 119(10):1270-80. Available from: http://onlinelibrary.wiley.com/ doi/10.1111/j.1471-0528.2012.03426.x/epdf. [Included in the review]

11. Bo K, Haakstad LAH. Is pelvic floor muscle training effective when taught in a general fitness class in pregnancy? A randomised controlled trial. Physiotherapy 2011; 97(3):190-5. [Included in the review]

12. Miquelutti MA, Cecatti JG, Makuch MY. Evaluation of a birth preparation program on lumbopelvic pain, urinary incontinence, anxiety and exercise: a randomized controlled trial. BMC Pregnancy and Childbirth 2013; 13:154. [Included in the review]
13. Eliasson K, Nordlander I, Larson B, Hammarstro $M$, Mattsson E. Influence of physical activity on urinary leakage in primiparous women. Scand J Med Sci Sports [internet] 2005 [cited 2014 oct 12]; 15(2):87-94. Available from: http://onlinelibrary. wiley.com/doi/10.1111/j.1600-0838.2004.407.x/ pdf. [Included in the review]

14. Sangsawang B, Serisathien Y. Effect of pelvic floor muscle exercise programme on stress urinary incontinence among pregnant women. J Adv Nurs. 2012; 68(9):1997-2007. [Included in the review]

15. Morkved S, Bo K, Schei B, Salvesen KA. Pelvic floor muscle training during pregnancy to prevent urinary incontinence: a single-blind randomized controlled trial. Obstet Gynecol. 2003; 101(2):3139. [Included in the review]

16. Butterfield YC, O'Connell B, Phillips D. Peripartum urinary incontinence: A study of midwives' knowledge and practices. Women and Birth 2007; 20(2): 65-9. [Included in the review]

17. Reilly ETC, Freeman RM, Waterfield MR, Waterfield $A E$, Steggles $P$, Pedlar F. Prevention of postpartum stress incontinence in primigravidae with increased bladder neck mobility: a randomised controlled trial of antenatal pelvic floor exercises. BJOG [internet] 2002 [cited 2014 oct 12]; 109(1):68-76. Available from: http://onlinelibrary. wiley.com/doi/10.1111/j.1471-0528.2002.t01-101116.x/epdf [Included in the review]

18. Low LK, Miller JM, Guo Y, Ashton-Miller JA, DeLancey JO, Sampselle CM. Spontaneous pushing to prevent postpartum urinary incontinence: a randomized, controlled trial. Int Urogynecol J. 2013; 24(3):453-60. [Included in the review]

19. Agur WI, Steggles P, Waterfield M, Freeman RM. The long-term effectiveness of antenatal pelvic floor muscle training: eight-year follow up of a randomised controlled trial. BJOG [internet] 2008 [cited 2014 oct 12]; 115(8):985-90. Available from: http://onlinelibrary.wiley.com/doi/10.1111/ j.1471-0528.2008.01742.x/epdf

20. Dumoulin C, Bourbonnais D, Morin M, Gravel D, Lemieux MC. Predictors of Success for Physiotherapy Treatment in Women With Persistent Postpartum Stress Urinary Incontinence. Archives of Physical Medicine and Rehabilitation, 2010; 91 (7): 1059-1063. 
21. Kocaöz S, Eroğlu K, Sivaslioğlu AA. Role of pelvic floor muscle exercises in the prevention of stress urinary incontinence during pregnancy and the postpartum period. Gynecologic and Obstetric Investigation 2013; 75 (1): 34-40.

22. Orawan L, Preecha W. Effect of pelvic floor muscle training (PFMT) during pregnancy on bladder neck descend and delivery. Journal of the Medical Association of Thailand 2014; 97:pp. S156-S163.

23. Reilly ETC, Freeman RM, Walterfield MR, Walterfield AE, Stegles $P$, Pedlar F. Prevention of postpartum stress incontinence in primigravidae with increased bladder neck mobility: a randomised controlled trial of antenatal pelvic floor exercises. BJOG [internet] 2012 [cited 2014 oct 12]; 109(1):68-76. Available from: http://onlinelibrary.wiley.com/doi/10.1111/j.1471-0528.2002. t01-1-01116.x/epdf
Todos os autores participaram das fases dessa publicação em uma ou mais etapas a seguir, de acordo com as recomendações do International Committe of Medical Journal Editors (ICMJE, 2013): (a) participação substancial na concepção ou confecção do manuscrito ou da coleta, análise ou interpretação dos dados; (b) elaboração do trabalho ou realização de revisão crítica do conteúdo intelectual; (c) aprovação da versão submetida. Todos os autores declaram para os devidos fins que são de suas responsabilidades o conteúdo relacionado a todos os aspectos do manuscrito submetido ao OBJN. Garantem que as questões relacionadas com a exatidão ou integridade de qualquer parte do artigo foram devidamente investigadas e resolvidas. Eximindo, portanto o OBJN de qualquer participação solidária em eventuais imbróglios sobre a materia em apreço. Todos os autores declaram que não possuem conflito de interesses, seja de ordem financeira ou de relacionamento, que influencie a redação e/ou interpretação dos achados. Essa declaração foi assinada digitalmente por todos os autores conforme recomendação do ICMJE, cujo modelo está disponível em http://www. objnursing.uff.br/normas/DUDE_final_13-06-2013.pdf

Recebido: $28 / 07 / 2015$

Revisado: $12 / 07 / 2015$

Aprobado: 26/01/2016 